\title{
Adaptación y Validación en Español de la Escala de Credibilidad en Profesores Universitarios
}

\author{
Adaptation and Validation in Spanish of the Source Credibility Measure in University \\ Professors
}

\author{
Facundo Froment ${ }^{1}$, M. Rocío Bohórquez ${ }^{2}$, Alfonso Javier García González ${ }^{3}$ y Eduardo García \\ Jiménez ${ }^{4}$
}

\section{Resumen}

El objetivo del estudio consistió en adaptar y validar al español la Escala de Credibilidad (McCroskey \& Teven, 1999) para medir la credibilidad de los profesores universitarios. Los participantes fueron 352 estudiantes de la Universidad de Sevilla pertenecientes a diversas titulaciones del área de las Ciencias Sociales. El análisis factorial exploratorio generó un modelo de tres factores alternativo al original. No obstante, tanto el modelo alternativo como el modelo original presentaron una adecuada consistencia interna no sólo en la escala global sino también en las tres subescalas; buenos índices de bondad de ajuste como confirmó el análisis factorial confirmatorio, apoyando así la estructura factorial de tres factores; invarianza por sexo y evidencia de validez concurrente con la motivación académica. Se concluye que el modelo original así como el modelo alternativo de la Escala de Credibilidad son instrumentos válidos y fiables para medir la credibilidad de los docentes universitarios.

Palabras clave: adaptación de escalas, validación, propiedades psicométricas, credibilidad docente, motivación académica

\begin{abstract}
The purpose of the study was to adapt to and validate to Spanish the Source Credibility Measure (McCroskey $\&$ Teven, 1999) to assess the credibility of university professors. The participants were 352 students from the University of Seville from different courses in the field of Social Sciences. The exploratory factor analysis generated an alternative three-factor model to the original one. However, both the alternative model and the original model presented adequate internal consistency not only in the global scale but also in the three subscales, good indexes of goodness of fit as demonstrated by the confirmatory factor analysis, supporting the factorial structure of three factors; invariance across sex and evidence of concurrent validity with academic motivation. It is concluded that the original model as well as the alternative model of the Source Credibility Measure are valid and reliable instruments to assess the credibility of university professors.
\end{abstract}

Keywords: adaptation of scales, validation, psychometric properties, teacher credibility, academic motivation

\footnotetext{
${ }^{1}$ Graduado en Pedagogía. Universidad de Sevilla. Doctorando en Educación. C/Pirotecnia S/N. 41013, Sevilla, España. Tel.: 666305944. Correo: facundopfroment@ hotmail.es

${ }^{2}$ Doctora en Psicología. Universidad de Sevilla. Profesora Ayudante Doctora. C/Pirotecnia S/N. 41013, Sevilla, España. Tel.: 955420489. Correo: rociobohorquez@us.es

${ }^{3}$ Doctor Europeo. Universidad de Sevilla. Profesor Titular. Decano de la Facultad de Ciencias de la Educación de la Universidad de Sevilla. C/Pirotecnia S/N. 41013, Sevilla, España. Tel.: 955420750. Correo: alfonsoj@us.es (Autor de correspondencia)

${ }^{4}$ Doctor en Educación. Universidad de Sevilla. Catedrático de Universidad. C/Pirotecnia S/N. 41013, Sevilla, España. Tel.: 955420644. Correo: egarji@us.es

Revista Iberoamericana de Diagnóstico y Evaluación - e Avaliação Psicológica. RIDEP · N51 Vol.2 · 61-76 2019

ISSN: 1135-3848 print /2183-6051online
} 


\section{Introducción}

La credibilidad es posiblemente el constructo que mayor atención ha recibido en el estudio de la comunicación humana (Teven \& Katt, 2016). Remontándonos a la Antigua Grecia, Aristóteles sostenía que la fuente de un mensaje es un factor determinante en la persuasión de ese mensaje debido a su influencia en cómo la audiencia recibe e interpreta el mensaje (Cooper, 1935). Es importante que los oradores aprendan a comunicarse de tal manera que los miembros de su audiencia les atribuyan credibilidad para poder así mejorar la persuasión del mensaje que se desea transmitir (Hovland, Janis, \& Kelley, 1954). En pocas palabras, la gente a menudo rechaza información de fuentes que carecen de credibilidad (Beatty \& Behnke, 1980). Por lo tanto, la credibilidad es un constructo dinámico al estar sujeto a cambios como resultado de experiencias que la audiencia tiene con una fuente particular (McCroskey, 1968).

Así, uno de los factores más importantes que afecta la relación profesor-alumno es la credibilidad del docente (Glascock \& Ruggiero, 2006; Myers, 2001; Teven, 2007); y puede definirse como la percepción del estudiante acerca de si el profesor es creíble o no (McCroskey, 1992). La credibilidad del profesor juega un papel determinante en las dinámicas de clase ya que las percepciones de la credibilidad del mismo impactan directamente en la comunicación profesor-alumno y, consecuentemente, en el proceso de enseñanza-aprendizaje (Finn et al., 2009; Russ, Simonds, \& Hunt, 2002). Así, constituye la variable primaria en la percepción del estudiante sobre el docente que tiene una influencia trascendental en el proceso de enseñanza-aprendizaje (McCroskey, Valencic, \& Richmond, 2004). De acuerdo con McCroskey y Teven (1999), está compuesta por tres dimensiones: competencia, confianza y buena voluntad. La competencia del docente hace referencia a la percepción del conocimiento o dominio del profesor en relación con la asignatura que imparte; los docentes competentes son considerados personas inteligentes, cualificadas, expertas e informadas. La confianza del docente se refiere a la percepción de fiabilidad y bondad del docente; los docentes que poseen confianza son considerados personas fiables, honestas, buenas y sinceras. La buena voluntad del docente consiste en el nivel en el que los estudiantes perciben que el profesor muestra interés por el bienestar del alumnado; los docentes que poseen buena voluntad son percibidos como personas comprensibles, empáticas, que tienen en cuenta las preocupaciones de los estudiantes y a los que les importan éstos (McCroskey \& Young, 1981; Frymier \& Thompson, 1992; McCroskey, 1992; Teven \& McCroskey, 1997).

La literatura existente acerca de la credibilidad del docente se ha centrado en analizar fundamentalmente los comportamientos $\mathrm{y}$ características propias del profesor que influyen en la percepción de credibilidad por parte de los estudiantes y en estudiar el impacto de la credibilidad del docente en los procesos de enseñanza-aprendizaje. En relación con las variables relacionadas con el comportamiento y las singularidades del docente, son percibidos como creíbles por los estudiantes los profesores que utilizan estrategias de búsqueda de afinidad con los estudiantes (Frymier \& Thompson, 1992); que se muestran cercanos (Johnson \& Miller, 2002; Pogue \& AhYun, 2006; Thweatt \& McCroskey, 1998); que emplean estilos comunicativos argumentativos (Schrodt, 2003; Edwards \& Myers, 2007), que se muestran asertivos y sensibles (Martin, Chesebro, \& Mottet, 1997); que utilizan la tecnología en la clase de forma moderada (Finn \& Ledbetter, 2013; Schrodt $\&$ Witt, 2006) y que revelan información personal relacionada con el contenido del curso y significativa para los estudiantes (Myers, Brann, $\&$ Members of Comm 600, 2009). Sin embargo, los docentes que son verbalmente agresivos (Mazer \& Stowe, 2016; Myers, 2001; Schrodt, 2003); los profesores que se comportan mal o exhiben conductas inapropiadas (Banfield, Richmond, \& McCroskey, 2006; Teven, 2007; Thweatt \& McCroskey, 1998) son menos creíbles. Asimismo, variables como la edad (Semlak \& Pearson, 2008); el sexo (Glascock \& Ruggiero, 2006; Patton, 1999; Wang, Novak, ScofieldSnow, Traylor, \& Zhou 2015); la raza (Glascock \& Ruggiero, 2006; Hendrix, 1998; Patton, 1999); la vestimenta (Lavin, Davies, \& Carr, 2010; Morris, Gorham, Cohen, \& Huffman, 1996; Sebastian \& Bristow, 2008); el atractivo físico 
(Wang et al., 2015) y la orientación sexual (Russ et al., 2002) del docente, influyen en la percepción de credibilidad por parte del alumnado.

En cuanto al impacto de la credibilidad del docente en los procesos de enseñanza-aprendizaje, cuando perciben al docente como creíble los estudiantes muestran mayor motivación académica (Frymier \& Thompson, 1992; Martin et al., 1997; Pogue \& AhYun, 2006; Tibbles, Richmond, McCroskey, \& Weber, 2008); mayor aprendizaje afectivo (Beatty \& Zahn, 1990; Henning, 2010; McCroskey et al., 2004; Teven, 2001; Teven \& McCroskey, 1997; Pogue \& AhYun, 2006; Tibbles et al., 2008); mayor aprendizaje cognitivo (McCroskey et al., 2004; Teven \& McCroskey, 1997; Tibbles et al., 2008); más satisfacción (Gaffney \& Gaffney, 2016; Teven \& Herring, 2005) y más empoderamiento (Ledbetter \& Finn, 2016; Schrodt et al., 2009). Igualmente, cuando el docente es creíble, los estudiantes se comunican más con el docente tanto dentro como fuera del aula (Myers, 2004; Nadler \& Nadler, 2001); se sienten mejor comprendidos por el profesor (Schrodt, 2003; Schrodt, Turman, \& Soliz, 2006); perciben más justicia en la clase (Chory, 2007); respetan más a los docentes (Martinez-Egger \& Powers, 2007) y evalúan positivamente a los profesores (Beatty \& Zahn, 1990; Teven \& McCroskey, 1997). No obstante, cuando el profesor carece de credibilidad, los alumnos exhiben comportamientos incívicos en el aula (Al-Zoubi, 2016; Miller, Katt, Brown, \& Sivo, 2014; Zhang, Zhang, \& Castelluccio, 2011).

Desde mediados de la década de 1960 hasta principios de la década de 1980, tuvieron lugar numerosas investigaciones centradas en desarrollar instrumentos para medir la credibilidad (Applbaum \& Anatol, 1973; Baudhuin \& Davis, 1972; McCroskey, 1966; McCroskey \& Young, 1981; Tuppen, 1974; Whitehead, 1968). En este sentido, Aristóteles postuló que la credibilidad estaba compuesta de tres elementos: inteligencia, carácter y buena voluntad (Cooper, 1935). Del mismo modo, Hovland et al. (1954) consideraron que la credibilidad de la fuente comprende tres elementos: competencia, confianza e intención hacia el receptor. De forma general, los teóricos coinciden en que hay una dimensión que puede ser referenciada como "competencia" y otra que puede ser referenciada como "confianza", de manera que en la mayoría de los estudios acerca de medición de la credibilidad se observaron dimensiones que podían denominarse "competencia" y "confianza", pero en ninguna se observó una dimensión que pudiera ser etiquetada apropiadamente como "buena voluntad" o "intención hacia el receptor" (McCroskey \& Teven, 1999). McCroskey y Young (1981) observaron que, cuando se sometían a técnicas de análisis factorial, sólo permanecían dos dimensiones de la credibilidad, siendo el tercer factor incluido junto a las otras dos dimensiones debido a que se habían desarrollado medidas satisfactorias para las dimensiones de "competencia" y "confianza", pero no para la dimensión "buena voluntad". Después de una reconceptualización y operacionalización de la dimensión de "buena voluntad", Teven y McCroskey (1997) reexaminaron el constructo de credibilidad, utilizando una escala bipolar de 10 ítems que incluía una dimensión de "buena voluntad", encontrando a través del análisis factorial que las percepciones de los estudiantes sobre la buena voluntad de sus profesores eran independientes de las otras dos dimensiones de credibilidad. Para replicar estos hallazgos fuera del ámbito académico, McCroskey y Teven (1999) examinaron la credibilidad de fuentes políticas, fuentes públicas y fuentes interpersonales. Utilizando el análisis factorial confirmatorio, hallaron nuevamente la estructura factorial de tres dimensiones del constructo de credibilidad, destacando además la fiabilidad y validez de la nueva dimensión de "buena voluntad". Así, desarrollaron y redefinieron el instrumento, resultando en una escala compuesta por 18 ítems que evalúan las tres dimensiones de la credibilidad: competencia, confianza y buena voluntad. De esta forma, McCroskey y Teven (1999) elaboraron el Source Credibility Measure para medir la credibilidad de una determinada fuente, empleándose principalmente en el ámbito académico para medir la credibilidad docente.

La escala ha sido utilizada igualmente para medir la credibilidad docente en otros países como Brasil (Ramos, Baghurst, \& McCullik, 2016) o Jordania (Al-Zoubi, 2016), así como en estudios comparados entre estudiantes estadounidenses y alumnos procedentes de Kenia 
(Johnson \& Miller, 2002); China (Zhang et al., 2011); China, Alemania y Japón (Zhang, 2009) y Brasil (Santilli, Miller, \& Katt, 2011). Según Okabe (1983), el significado de credibilidad puede diferir de una cultura a otra, de modo que las atribuciones de cada cultura acerca de la credibilidad de una fuente pueden asimismo variar. Así, King, Minami y Samovar (1985) descubrieron que los japoneses perciben la credibilidad de la fuente de manera diferente a la de los estadounidenses ya que la muestra estadounidense produjo dos factores, mientras que la muestra japonesa generó cuatro factores. Del mismo modo, Heyman (1992) determinó que la percepción de los australianos de la credibilidad de la fuente era ligeramente diferente a la de los singapurenses, generándose en la muestra australiana dos dimensiones mientras que en la muestra singapurense se produjo un único factor. No obstante, Yoon, Kim y Kim (1998) encontraron que las tres dimensiones de credibilidad de la fuente son igualmente aplicables a muestras estadounidenses y coreanas. Estos hallazgos algo inconsistentes pueden deberse a una limitación metodológica debido a que los estudios anteriormente citados se basaron en análisis factoriales exploratorios para probar las dimensiones (Zhang, 2009). En este sentido, centrándonos exclusivamente en la medición de la credibilidad docente, Zhang (2009) confirmó en su estudio transcultural a través del análisis factorial confirmatorio la estructura factorial de tres dimensiones de la escala, de modo que sus resultados apuntan a que la Source Credibility Measure mantiene su estructura factorial de tres dimensiones al aplicarla para medir la credibilidad docente con muestra de otros países y culturas. Debido a la ausencia de un instrumento válido y fiable en español que permita medir la credibilidad docente, el objetivo del presente es realizar una evaluación de las propiedades psicométricas de la Source Credibility Measure traducida al español, probando su validez y fiabilidad, su estructura factorial de tres dimensiones y la invarianza de la misma según el sexo.

\section{Método}

\section{Participantes}

Para la selección de los participantes se aplicó un diseño muestral no probabilístico por accesibilidad (Gil-Escudero \& Martínez-Arias, 2001). La muestra estuvo compuesta por 352 estudiantes de la Universidad de Sevilla de los Grados en Educación Primaria, Psicología, Relaciones Laborales y Recursos Humanos y Doble Grado en Finanzas y Contabilidad y Relaciones Laborales y Recursos Humanos. Los criterios de inclusión fueron carreras del ámbito de las Ciencias Sociales y asignaturas con programas docentes similares. La edad media de los participantes fue de 19.79 (DT=3.09), siendo la distribución de la participación por sexo de 87 hombres (24.7\%) y 265 mujeres (75.3\%).

\section{Instrumentos}

Para medir la credibilidad docente se empleó la mencionada Source Credibility Measure de McCroskey y Teven (1999). La escala presenta 18 adjetivos bipolares, seis para cada dimensión, distribuidos de la siguiente manera (Tabla 1):

Tabla 1. Dimensiones e ítems de la Escala de Credibilidad

\begin{tabular}{lc}
\hline Dimensiones & Ítems \\
\hline Competencia & $\begin{array}{c}\text { Inteligente }- \text { Poco inteligente; Poco } \\
\text { formado - Formado; Inexperto - } \\
\text { Experto; Informado - Desinformado; } \\
\text { Incompetente - Competente; } \\
\text { Inteligente - Idiota }\end{array}$ \\
Buena & Le importo - No le importo; Tiene \\
Voluntad & en cuenta mis intereses - No tiene en \\
& No enta mis intereses; Egocéntrico - \\
& - No se preocupa por mí; Insensible \\
& - Sensible; No comprensivo - \\
& Comprensivo \\
Confianza & Fiable; Honorable - Deshonroso; \\
& Moral - Inmoral; Poco ético- Ético; \\
& Falso - Sincero \\
\hline
\end{tabular}

Para completar la escala, el alumno debe indicar su percepción del profesor de acuerdo a valores que van desde el 1 hasta el 7, teniendo en cuenta que cuanto más cerca esté el número del adjetivo, mayor certeza habrá en la evaluación del profesor. De acuerdo a la consistencia interna del instrumento, se obtuvo en el estudio original un valor de alpha de Cronbach para la escala global de .94 y los siguientes valores de alpha de Cronbach para las tres dimensiones: Competencia .78; Confianza .92 y Buena Voluntad 89.

Asimismo, para evaluar la validez concurrente 
de la escala de credibilidad, se utilizó el Cuestionario de Estrategias de Aprendizaje y Motivación (Martínez \& Galán, 2000). Este instrumento está compuesto por dos cuestionarios, uno que evalúa la motivación y otro que evalúa las estrategias de aprendizaje de estudiantes universitarios, seleccionando para este estudio únicamente el cuestionario de motivación académica. Aunque la motivación académica depende en parte de factores personales y socioculturales, es el docente quien tiene un papel fundamental en fomentar la motivación académica del alumnado (Gómez-López, Granero-Gallegos, Baena-Extremera, Bracho-Amador, \& PérezQuero, 2015; Leal-Soto, \& Alonso-Tapia, 2017).

El cuestionario de motivación académica está compuesto por 31 ítems, distribuidos en las siguientes subescalas: Orientación Intrínseca (cuatro ítems), Orientación Extrínseca (cuatro ítems), Valor de la tarea (seis ítems), Control sobre creencias (cuatro ítems), Autoeficacia (ocho ítems) y Ansiedad (cinco ítems). Para responder a los ítems, el estudiante debe escoger entre valores que van desde el 1 (No me describe en absoluto) hasta el 7 (Me describe totalmente). Este instrumento fue sometido a un análisis de fiabilidad en el que se obtuvieron los siguientes valores: .99 en la escala global; .93 en Orientación Intrínseca; .91 en Orientación Extrínseca, .95 en Valor de la tarea; .90 en Control sobre creencias; .96 en Autoeficacia; y, .93 en Ansiedad. En consecuencia, podemos afirmar que los valores obtenidos son aceptables y, por lo tanto, la escala es fiable.

\section{Procedimiento}

Para el proceso de adaptación de la escala Source Credibility Measure al español se han seguido los criterios planteados por Hambleton (2004), quien propone la necesidad de asegurar la equivalencia de concepto, lingüística y métrica de las adaptaciones de instrumentos de medida. La adaptación de la escala fue realizada utilizando un proceso de retrotraducción: la traducción inicial realizada por dos profesionales de lengua inglesa cuya lengua materna era el español y la traducción inversa realizada por dos traductores bilingües cuya lengua nativa era el inglés. Ambas traducciones no presentaron discrepancias, por lo que no fue necesario modificar los ítems, logrando así la adaptación final en español de la Escala de Credibilidad. En cuanto al procedimiento de administración de los instrumentos, los participantes completaron voluntariamente los dos instrumentos, brindando todos ellos consentimiento informado previo a la cumplimentación de los mismos. Asimismo, se les explicó los objetivos del estudio y se enfatizó el carácter anónimo de la participación. Del mismo modo, se destacó que los datos recogidos serían utilizados únicamente con fines de investigación y se pidió sinceridad al responder, señalando que no hay respuestas correctas o incorrectas. La escala y el cuestionario se administraron en formato de lápiz y papel por expertos en el aula siguiendo el siguiente orden: Escala de Credibilidad y Cuestionario de Motivación Académica. Los participantes dispusieron de 25 minutos para completar ambos instrumentos. Los datos recogidos fueron procesados en una base de datos para su posterior análisis.

\section{Análisis de datos}

Se analizó la escala Source Credibility Measure (McCroskey \& Teven, 1999) en su versión original de 18 ítems, estudiándose su estructura a través de un análisis factorial exploratorio. Ateniendo al estudio original, el método de extracción de factores utilizado para analizar los datos de nuestro estudio fue el de componentes principales con rotación promax al existir correlación entre las dimensiones, requiriendo asimismo la extracción de tres factores. Los tres factores obtenidos así como la escala completa fueron sometidos a un procedimiento de análisis de fiabilidad mediante el estadístico alfa de Cronbach y omega de McDonald para la valoración de la consistencia interna de la escala.

Para constatar la validez del modelo se realizó un análisis factorial confirmatorio, empleándose el procedimiento de mínimos cuadrados no ponderados para la estimación de los parámetros. La elección de este método de estimación se debe a que no requiere que las variables sigan una distribución determinada (Batista \& Coenders, 2000; Brown, 2006). Posteriormente, se analizó la invarianza del modelo según el sexo $\mathrm{y}$, finalmente, se calcularon correlaciones de Spearman entre la escala de credibilidad con 
motivación académica para evaluar la validez concurrente del modelo.

\section{Resultados}

\section{Análisis factorial exploratorio}

Con respecto al análisis factorial exploratorio, en la medida Kaiser-Meyer-Olkin de adecuación de muestreo (KMO) se obtuvo un valor de .94, superando el valor recomendado de $.60 \mathrm{y}$ en la prueba de esfericidad de Bartlett se obtuvo un valor de .0001 $(p<.01)$, siendo ambos valores aceptables, lo que indica que la matriz de correlación no es una matriz de identidad, por lo que es apropiado llevar a cabo el análisis factorial. Una vez realizado el análisis de los componentes principales fueron extraídos los tres factores requeridos, que obtuvieron valores propios superiores a la unidad $(9.19,1.76$ y 1.01$)$, ascendiendo la varianza total explicada en el conjunto de la escala a un $66.53 \%$ (un $51.10 \%$ en el primer factor, un $9.78 \%$ en el segundo factor y un $5.64 \%$ en el tercer factor).

Como se puede observar en la matriz de componentes rotados (promax con normalización Kaiser), la primera dimensión "Competencia" incluye desde el ítem 1 al 6; la segunda dimensión "Buena Voluntad" incluye los ítems 7, 8 y 10 y la tercera dimensión "Confianza" incluye el ítem 9 y desde el ítem 11 al 18 (Tabla 2).

\section{Análisis de fiabilidad}

Este modelo, obtenido en el análisis factorial exploratorio, fue sometido a un análisis de su fiabilidad. El alfa de Cronbach obtenido para el conjunto de la escala fue de .94. En el análisis de fiabilidad de cada uno de los factores se obtuvo un valor de .87 para el primer factor (Competencia); .90 para el segundo factor (Buena Voluntad) y .91 para el tercer factor (Confianza). El cálculo del coeficiente omega de McDonald (1999) nos aporta un valor más estable dado que se obtiene a partir de los pesos factoriales, es decir, de la suma ponderada de las variables estandarizadas. Los valores de ambos coeficientes se recogen en la Tabla 3 y nos permitirían afirmar que los valores obtenidos son aceptables y, por lo tanto, la escala es fiable.
Tabla 2. Matriz de componentes rotados del modelo obtenido del análisis factorial exploratorio

\begin{tabular}{lccc}
\hline Escala & Factor 1 & Factor 2 & Factor 3 \\
\hline Inteligente - Poco & .828 & & \\
inteligente & .8 & & \\
Poco formado - & .720 & & \\
Formado & & & \\
Inexperto - & .811 & & \\
Experto & & & \\
Informado - & .772 & & \\
Desinformado & & & \\
Incompetente - & .766 & & \\
Competente & & & \\
Inteligente - & .824 & & \\
Idiota & & \\
Le importo - No & & .899 & \\
le importo & & & \\
Tiene en cuentas & & & \\
mis intereses - & & .908 & \\
No tiene en & & \\
cuenta mis & & & \\
intereses & & & \\
Egocéntrico - No & & & .728 \\
egocéntrico & & & \\
Se preocupa por & & .903 & \\
mí - No se & & .803 \\
preocupa por mí & & & \\
Insensible - & & & .700 \\
Sensible & & .801 \\
No comprensivo & & & .746 \\
- Comprensivo & & & .817 \\
Honesto - & & & \\
Deshonesto & & & \\
Poco fiable - & & & .841 \\
Fiable & & \\
Honorable - & & & \\
Deshonroso & & & \\
Moral - Inmoral & & & \\
Poco ético - Ético & & \\
Falso - Sincero & & & \\
\hline
\end{tabular}

Tabla 3. Estadísticas de fiabilidad del modelo obtenido en el análisis factorial exploratorio

\begin{tabular}{lccc}
\hline & $\begin{array}{c}\text { alfa de } \\
\text { Cronbach }\end{array}$ & $\begin{array}{c}\text { omega de } \\
\text { McDonald }\end{array}$ & $\begin{array}{c}\mathrm{N}^{\circ} \mathrm{de} \\
\text { elementos }\end{array}$ \\
\hline $\begin{array}{l}\text { Escala } \\
\text { completa }\end{array}$ & .941 & .969 & 18 \\
$\begin{array}{l}\text { Primer } \\
\text { factor }\end{array}$ & .879 & .905 & 6 \\
$\begin{array}{l}\text { Segundo } \\
\text { factor }\end{array}$ & .906 & .948 & 3 \\
$\begin{array}{l}\text { Tercer } \\
\text { factor }\end{array}$ & .918 & .930 & 9 \\
\hline
\end{tabular}

\section{Análisis factorial confirmatorio}

Este modelo de tres factores con seis ítems en el primer factor, tres ítems en el segundo factor y nueve ítems en el tercer factor, extraído del análisis factorial exploratorio, fue sometido a un análisis factorial confirmatorio. Se empleó el método de mínimos cuadrados no ponderados 


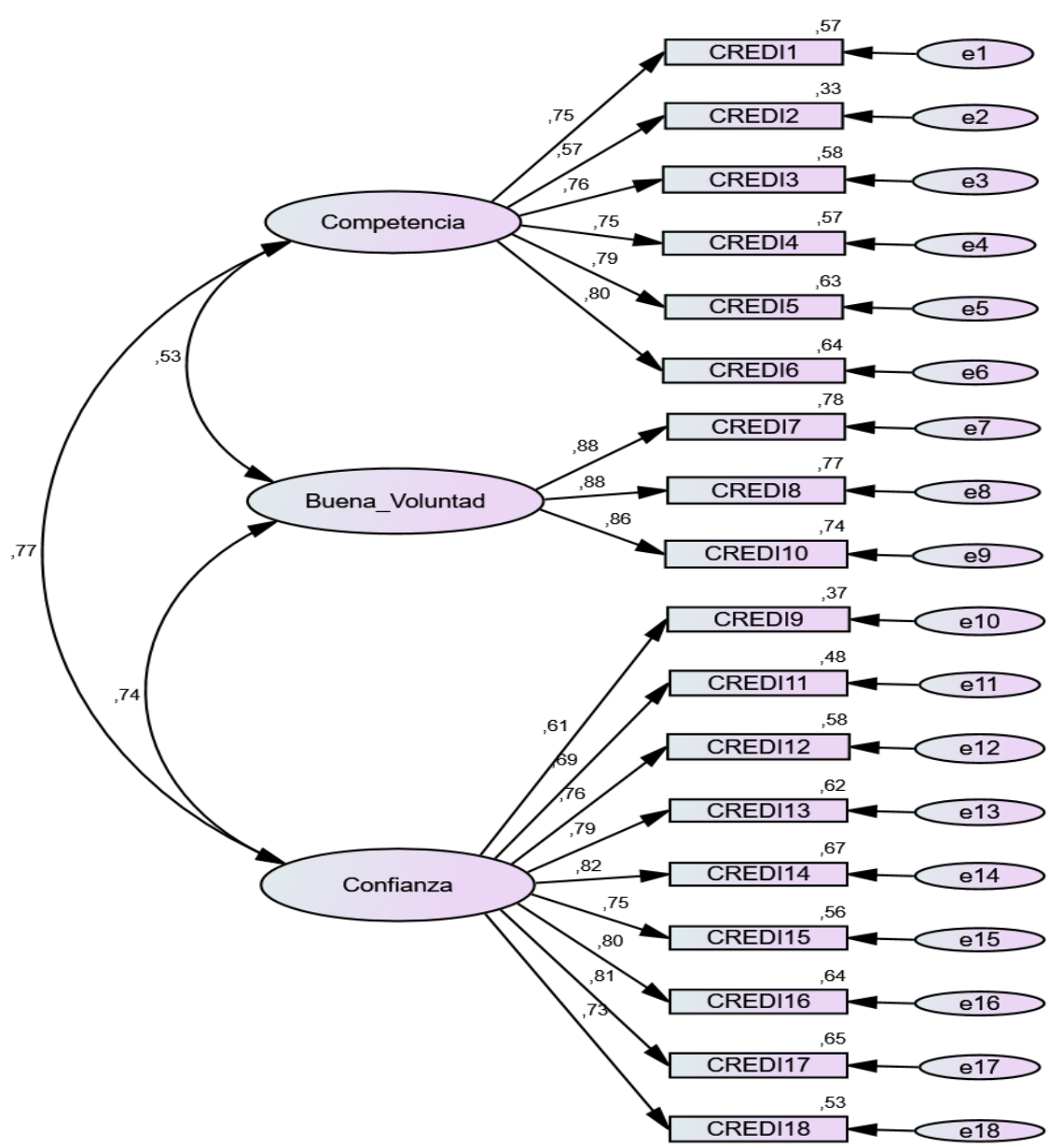

Figura 1. Estimaciones estandarizadas del modelo obtenido del análisis factorial exploratorio

para la estimación de los parámetros. En la Figura 1 se aprecian los parámetros estimados estandarizados del modelo, donde se observa cómo el peso factorial de los dieciocho ítems alcanza valores elevados, siendo el menor .57 correspondiente al Ítem 2.

Para evaluar la bondad de ajuste del modelo se escogieron la raíz cuadrada media residual (RMR), el índice de bondad de ajuste (GFI) y el índice de bondad de ajuste corregido (AGFI). Se consideran aceptables valores del RMR por debajo de .08 (Browne \& Cudeck, 1992; Hu \& Bentler, 1999) y valores del GFI y del AGFI por encima de .90 (Byrne, 2016; Schumacher \& Lomax, 2004). Cabe señalar que no se consideró el chi-cuadrado como indicador de ajuste ya que es muy sensible al tamaño muestral y está fundamentada en la distribución central de $\chi^{2}$.

Los resultados del análisis factorial confirmatorio indican que el modelo obtenido del análisis factorial exploratorio presenta un valor de RMR de .054, un valor de GFI de .99 y de AGFI de .99 , de modo que el modelo contiene buenos índices de ajuste. Asimismo, para constatar una vez más la bondad de ajuste del modelo, se realizó una análisis factorial confirmatorio del mismo empleando correlaciones policóricas en lugar de correlaciones de Pearson; de hecho, el método de mínimos cuadrados no ponderados se basa en la matriz de correlaciones policóricas. Cuando las variables son ordinales se recomiendan emplear en los análisis factoriales confirmatorios las correlaciones policóricas ya que reproducen de manera más precisa la bondad de ajuste de los modelos en comparación con las correlaciones de Pearson (DiStefano, 2002; Holgado-Tello, Chacón-Moscoso, Barbero-García, \& Vila-Abad, 2010). Los resultados del análisis factorial confirmatorio mediante correlaciones policóricas señalan que el modelo generado a partir del análisis factorial exploratorio presenta un valor de RMR de .007, un valor de GFI de 1.00 y un valor de AGFI de 1.00, de modo que el modelo obtiene también buenos índices de ajuste. Por último, se llevó a cabo la invarianza del modelo de acuerdo al sexo, obteniéndose un valor de RMR de .064, 
Tabla 4. Correlación entre la escala obtenida del análisis factorial exploratorio y motivación académica

\begin{tabular}{lcccc}
\hline Variables & Competencia & Buena Voluntad & Confianza & Credibilidad \\
\hline Motivación académica & $.36^{* *}$ & $.36^{* * *}$ & $.43^{* *}$ & $.46^{* * *}$ \\
O. Intrínseca & $.28^{* *}$ & $.25^{* *}$ & $.34^{* *}$ & $.34^{* *}$ \\
O. Extrínseca & $.20^{* *}$ & $.20^{* *}$ & $.23^{* *}$ & $.25^{* *}$ \\
Valor de la tarea & $.39^{* *}$ & $.38^{* *}$ & $.44^{* *}$ & $.47^{* *}$ \\
Control sobre creencias & $.29^{* *}$ & $.29^{* *}$ & $.34^{* *}$ & $.36^{* *}$ \\
Autoeficacia & $.31^{* *}$ & $.34^{* *}$ & $.46^{* *}$ & $.45^{* *}$ \\
Ansiedad & -.07 & -.09 & $-.15^{* *}$ & $-.13^{*}$ \\
Media & 36.30 & 14.79 & 50.97 & 102.06 \\
DS & 4.64 & 3.61 & 7.91 & 14.22 \\
\hline Nota $* p<.05 . *^{* *} p<.01$. DS=Desviación estándar & & &
\end{tabular}

un valor de GFI de .99 y un valor de AGFI de .98, lo que indica que el modelo presenta asimismo buenos índices de ajuste y que no existen diferencias por sexo en la escala.

\section{Evidencias de validez concurrente}

Para evaluar la validez concurrente del modelo, se calcularon correlaciones de Spearman entre la escala de credibilidad docente obtenida del análisis factorial exploratorio y sus tres subescalas (competencia, buena voluntad y confianza) con motivación académica y sus seis subescalas (orientación intrínseca, orientación extrínseca, valor de la tarea, control sobre creencias, autoeficacia y ansiedad). Como se aprecia en la Tabla 4, la escala de credibilidad docente obtenida del análisis factorial exploratorio correlaciona positivamente con la motivación académica $(r=.46, p<.01)$. Asimismo, correlaciona positivamente con orientación intrínseca $(r=.34$, $p<.01)$, orientación extrínseca $(r=.25, p<.01)$, valor de la tarea $(r=.47, p<.01)$, control sobre creencias $(r=.36, p<.01)$ y autoeficacia $(r=.45$, $p<.01)$ y correlaciona negativamente con ansiedad $(r=-.13, \quad p<.05)$. La competencia correlaciona positivamente con orientación intrínseca $(r=.28$, $p<.01)$, orientación extrínseca $(r=.20, p<.01)$, valor de la tarea $(r=.39, p<.01)$, control sobre creencias $(r=.29, p<.01)$ y autoeficacia $(r=.31, p<.01)$. La buena voluntad correlaciona positivamente con orientación intrínseca $(r=.25, p<.01)$, orientación extrínseca $(r=.20, p<.01)$, valor de la tarea $(r=.38$, $p<.01)$, control sobre creencias $(r=.29, p<.01)$ y autoeficacia $(r=.34, p<.01)$. La confianza correlaciona positivamente con orientación intrínseca $(r=.34$, $p<.01)$, orientación extrínseca $(r=.23, p<.01)$, valor de la tarea $(r=.44, p<.01)$, control sobre creencias $(r=.34, p<.01)$, y autoeficacia $(r=.46, p<.01)$ y correlaciona negativamente con ansiedad $(\mathrm{r}=-.15$, $p<.01)$.
No obstante, cabe destacar que este modelo obtenido del análisis factorial exploratorio constituye un modelo diferente al original ya que a pesar de que se respeta la estructural factorial de tres factores y contiene buenos índices de ajuste como se ha demostrado en el análisis factorial confirmatorio, este modelo presenta seis ítems en el primer factor, tres ítems en el segundo factor y nueve ítems en el tercer factor mientras que el modelo original presenta 6 ítems en cada uno de los tres factores. Es decir, el análisis factorial exploratorio ha generado un modelo alternativo que si bien difiere del modelo original presenta unos índices de ajuste aceptables. Sin embargo, se consideró apropiado evaluar las propiedades psicométricas del modelo original llevando a cabo para ello los mismos procedimientos de análisis de datos que los empleados en el modelo obtenido del análisis factorial exploratorio.

Tabla 5. Estadísticas de fiabilidad de la escala

\begin{tabular}{lccc}
\multicolumn{4}{c}{ original } \\
\hline & $\begin{array}{c}\text { alfa de } \\
\text { Cronbach }\end{array}$ & $\begin{array}{c}\text { omega de } \\
\text { McDonald }\end{array}$ & $\begin{array}{c}\mathrm{N}^{\mathbf{0}} \text { de } \\
\text { elementos }\end{array}$ \\
\hline Escala completa & .941 & .964 & 18 \\
Primer factor & .879 & .905 & 6 \\
Segundo factor & .876 & .878 & 6 \\
Tercer factor & .909 & .914 & 6 \\
\hline
\end{tabular}

\section{Análisis de fiabilidad del modelo original}

En relación con los análisis de fiabilidad, como se ha mencionado previamente, el alfa de Cronbach obtenido para el conjunto de la escala fue de .94 . En el análisis de fiabilidad de cada uno de los factores de la escala original se obtuvo un valor de .87 para el primer factor (Competencia); .87 para el segundo factor (Buena Voluntad) y .90 para el tercer factor (Confianza), por lo que podemos afirmar que los valores obtenidos en el modelo original son aceptables y, por lo tanto, la escala es fiable (Tabla 5). 


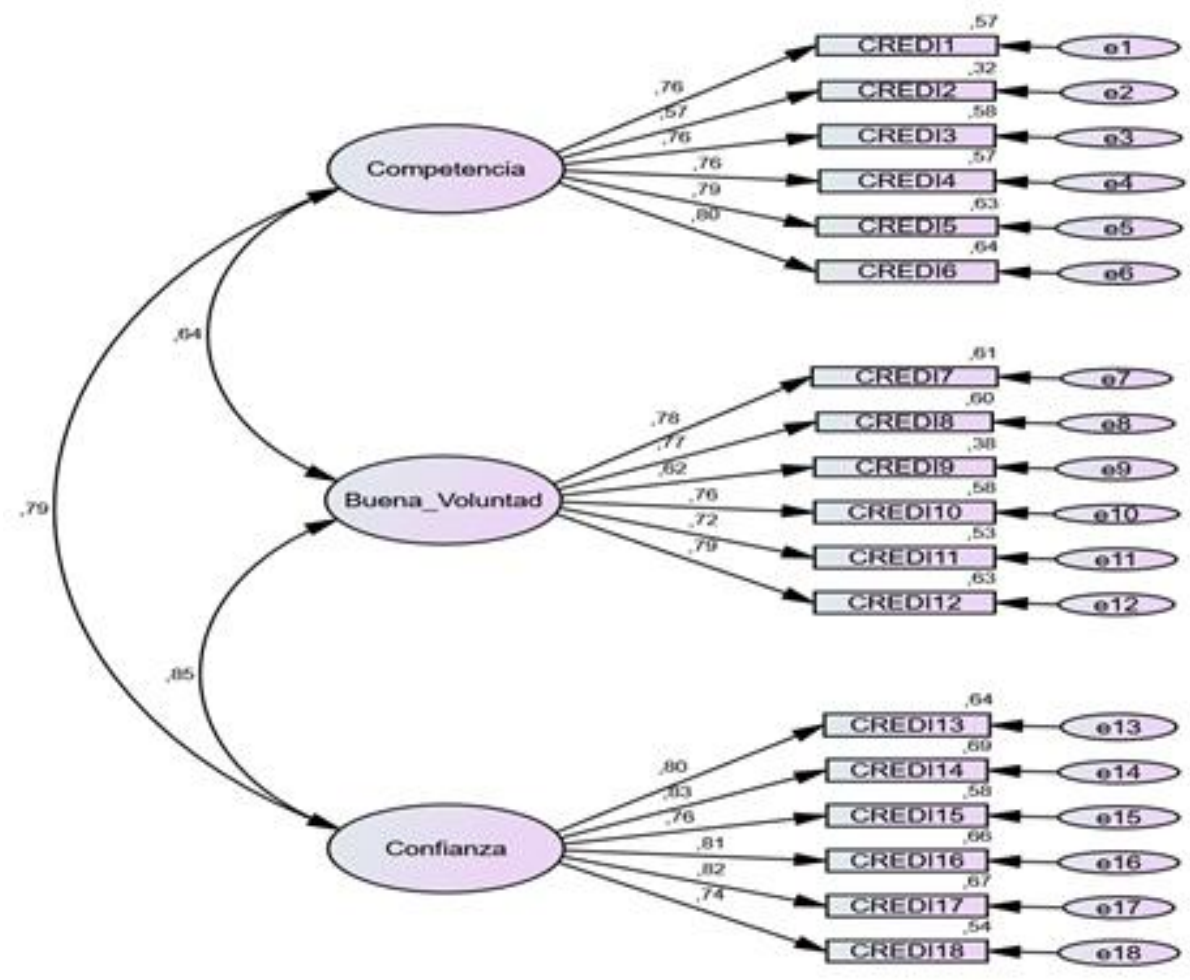

Figura 2. Estimaciones estandarizadas del modelo original

\section{Análisis factorial confirmatorio del modelo original}

Este modelo original de tres factores con seis ítems en cada uno de los tres factores fue sometido a un análisis factorial confirmatorio. Se empleó el método de mínimos cuadrados no ponderados para la estimación de los parámetros. En la Figura 2 se aprecian los parámetros estimados estandarizados del modelo original, donde se observa cómo el peso factorial de los dieciocho ítems alcanza valores elevados, siendo el menor .57 correspondiente al Ítem 2.

Para evaluar la bondad de ajuste del modelo original se escogieron nuevamente la raíz cuadrada media residual (RMR), el índice de bondad de ajuste (GFI) y el índice de bondad de ajuste corregido (AGFI), excluyendo una vez más el chi-cuadrado debido a su sensibilidad hacia el tamaño muestral. Los resultados del análisis factorial confirmatorio indican que el modelo original presenta un valor de RMR de .073, un valor de GFI de .98 y de AGFI de .98 , de modo que el modelo obtiene índices de ajuste aceptables. Igualmente, para constatar nuevamente la bondad de ajuste del modelo original, se realizó una análisis factorial confirmatorio del modelo empleando correlaciones policóricas en lugar de correlaciones de Pearson. Los resultados del análisis factorial confirmatorio del modelo original mediante correlaciones policóricas señalan que el modelo presenta un valor de RMR de .008, un valor de GFI de 1.00 y un valor de AGFI de 1.00, de modo que el modelo obtiene igualmente buenos índices de ajuste. Por último, se llevó a cabo la invarianza del modelo original de acuerdo al sexo, obteniéndose un valor de RMR de .077, un valor de GFI de .98 y un valor de AGFI de .98, lo que indica que el modelo original presenta buenos índices de ajuste y que no existen diferencias por sexo en la escala.

\section{Evidencias de validez concurrente del modelo original}

Para evaluar la validez concurrente del modelo, se calcularon correlaciones de Spearman entre la escala original de credibilidad docente y sus tres subescalas (competencia, buena voluntad y confianza) con motivación académica y sus seis subescalas (orientación intrínseca, orientación extrínseca, valor de la tarea, control sobre creencias, autoeficacia y ansiedad). Como se aprecia en la Tabla 6, la escala original de credibilidad docente correlaciona positivamente con la motivación académica $(r=.46, p<.01)$. 
Tabla 6. Correlación entre la escala original y motivación académica

\begin{tabular}{lcccc}
\hline Variables & Competencia & Buena Voluntad & Confianza & \multicolumn{1}{c}{ Credibilidad } \\
\hline Motivación académica & $.36^{* *}$ & $.39^{* * *}$ & $.45^{* * *}$ & $.46^{* *}$ \\
O. Intrínseca & $.28^{* *}$ & $.28^{* *}$ & $.35^{* * *}$ & $.34^{* *}$ \\
O. Extrínseca & $.20^{* *}$ & $.22^{* *}$ & $.24^{* *}$ & $.25^{* *}$ \\
Valor de la tarea & $.39^{* *}$ & $.42^{* *}$ & $.44^{* *}$ & $.47^{* *}$ \\
Control sobre creencias & $.29^{* *}$ & $.32^{* *}$ & $.35^{* *}$ & $.36^{* *}$ \\
Autoeficacia & $.31^{* *}$ & $.41^{* *}$ & $.46^{* *}$ & $.45^{* *}$ \\
Ansiedad & -.07 & $-.13^{*}$ & $-.12^{*}$ & $-.13^{*}$ \\
Media & 36.30 & 31.26 & 34.50 & 102.06 \\
DS & 4.64 & 5.97 & 5.39 & 14.22 \\
\hline Nota $* p<.05 . * * p<01$. DS=Desviación estándar & & &
\end{tabular}

Igualmente, correlaciona positivamente con orientación intrínseca $(r=.34, p<.01)$, orientación extrínseca $(r=.25, p<.01)$, valor de la tarea $(r=.47$, $p<.01)$, control sobre creencias $(r=.36, p<.01) \mathrm{y}$ autoeficacia $(r=.45, \quad p<.01) \quad \mathrm{y}$ correlaciona negativamente con ansiedad $(r=-.13, p<.05) . \mathrm{La}$ competencia correlaciona positivamente con orientación intrínseca $(r=.28, p<.01)$, orientación extrínseca $(r=.20, p<.01)$, valor de la tarea $(r=.39$, $p<.01)$, control sobre creencias $(r=.29, p<.01) \mathrm{y}$ autoeficacia $(r=.41, p<.01)$. La buena voluntad correlaciona positivamente con orientación intrínseca $(r=.28, p<.01)$, orientación extrínseca $(r=.22, p<.01)$, valor de la tarea $(r=.42, p<.01)$, control sobre creencias $(r=.32, \quad p<.01) \quad \mathrm{y}$ autoeficacia $(r=.41, \quad p<.01)$ y correlaciona negativamente con ansiedad $(r=-.13, p<.05)$. La confianza correlaciona positivamente con orientación intrínseca $(r=.35, p<.01)$, orientación extrínseca $(r=.24, p<.01)$, valor de la tarea $(r=.44, p<.01)$, control sobre creencias $(r=.35$, $p<.01) \quad$ y autoeficacia $(r=.46, \quad p<.01) \quad$ y correlaciona negativamente con ansiedad $(r=-.12$, $p<.05)$.

\section{Discusión}

El objetivo del presente estudio consistió en traducir al español y evaluar las propiedades psicométricas de la escala Source Credibility Measure, un instrumento que permite medir la credibilidad de una determinada fuente, siendo utilizada fundamentalmente en el ámbito académico para evaluar, como en este estudio, la credibilidad del profesorado. Esta es la primera vez que se ha empleado la escala utilizando una muestra hispanohablante. De acuerdo con los resultados obtenidos, tanto el modelo original como el modelo alternativo presentan una estructura de tres factores, una fiabilidad aceptable y evidencia adecuada de validez.

En relación con la consistencia interna, en la escala original se obtuvo un valor de alfa de Cronbach para la escala global de .94; .87 para Competencia; .87 para Buena Voluntad y .90 para Confianza, mientras que en el modelo alternativo se obtuvo un valor de Alfa de Cronbach para la escala global de $.94 ; .87$ para Competencia; .90 para Buena Voluntad y .91 para Confianza. Cabe destacar que la consistencia interna obtenida de la muestra española para ambos modelos fue similar a la obtenida por los autores originales (.94 para la escala global; .78 para Competencia; .89 para Buena Voluntad y .92 para Confianza) (McCroskey \& Teven, 1999). Del mismo modo, la alta fiabilidad de la escala obtenida de la muestra española coincide con la consistencia interna de la escala Source Credibility Measure de diversas investigaciones que la emplearon en diferentes países y culturas: .85 para la escala global con estudiantes kenianos (Johnson \& Miller, 2002); .71 para Competencia, .75 para Buena voluntad y .89 para Confianza con estudiantes brasileños (Santilli et al., 2011); .74 para Competencia, .75 para Buena Voluntad y .69 para Confianza con estudiantes jordanos (Al-Zoubi, 2016); .92 para la escala global, .90 para Competencia, .72 para Buena Voluntad y .90 para Confianza con estudiantes chinos (Zhang et al., 2011) y .92 para la escala global, .87 para Competencia, .82 para Buena Voluntad y .81 para Confianza con estudiantes alemanes y .93 para la escala global, .85 para Competencia, .80 para Buena Voluntad y .85 para Confianza con estudiantes japoneses (Zhang, 2009). En cuanto a la estructura factorial de la escala, el análisis factorial exploratorio generó un modelo de tres factores alternativo al original que fue sometido a un análisis factorial 
confirmatorio. Los resultados del análisis factorial confirmatorio apoyaron la estructura factorial del modelo alternativo, obteniéndose índices de bondad de ajuste adecuados (RMR=.054; $\mathrm{GFI}=.99 ; \mathrm{AGFI}=.99$ ). Este modelo alternativo fue sometido asimismo a un análisis factorial confirmatorio empleándose correlaciones policóricas en lugar de correlaciones de Pearson, obteniéndose igualmente índices de bondad de ajuste adecuados (RMR $=.007 ; \quad$ GFI $=1.00$; AGFI=1.00). Igualmente, se realizó la invarianza del modelo alternativo atendiendo al sexo de los participantes, obteniéndose índices de bondad de ajuste adecuados (RMR =.064; $\quad$ GFI=.99; AGFI=.98), de modo que no existen diferencias por sexo en la escala. La obtención de un modelo alternativo al original coincide con los estudios de Heyman (1992) y King et al. (1985), quienes obtuvieron asimismo modelos diferentes al original que, sin embargo, presentaban índices de bondad de ajuste aceptables con muestras australianas y singapurenses y con muestras japonesas respectivamente. La obtención de un modelo diferente al original puede deberse a que, como afirma Okabe (1983), el concepto de credibilidad puede diferir de una cultura a otra, por lo que las atribuciones de cada cultura con respecto a la credibilidad pueden variar.

Tras la obtención de un modelo alternativo, se evaluaron las propiedades psicométricas del modelo original. El modelo original fue sometido a un análisis factorial confirmatorio cuyos resultados apoyaron su estructura factorial, obteniéndose índices de bondad de ajuste adecuados (RMR=.073; GFI=.98; AGFI=.98). El modelo original fue sometido igualmente a un análisis factorial confirmatorio empleándose correlaciones policóricas en lugar de correlaciones de Pearson, obteniéndose asimismo índices de bondad de ajuste adecuados (RMR=.008; GFI=1.00; AGFI=1.00). Del mismo modo, se realizó la invarianza del modelo original de acuerdo al sexo de los participantes, obteniéndose índices de bondad de ajuste adecuados (RMR=.077; GFI=.98; AGFI=.98), por lo que no existen diferencias por sexo en la escala. Estos resultados coinciden con Zhang (2009) quien confirmó a través de un análisis factorial confirmatorio la estructura factorial de tres dimensiones de la escala Source Credibility
Measure para medir la credibilidad del profesorado mediante una muestra de estudiantes chinos, alemanes y japoneses.

Con respecto a la validez de los modelos, se ha encontrado evidencia de validez concurrente de ambos modelos con la motivación académica y sus seis subescalas (orientación intrínseca, orientación extrínseca, valor de la tarea, control sobre creencias, autoeficacia y ansiedad). Tanto el modelo original de credibilidad como el alternativo correlacionan positivamente con la motivación académica, coincidiendo así con estudios previos que destacan una correlación positiva entre credibilidad docente y motivación del estudiante (Frymier \& Thompson, 1992; Martin et al., 1997; Pogue \& AhYun, 2006; Tibbles et al., 2008).

Asimismo, en el modelo original, la escala global de credibilidad correlaciona positivamente con orientación intrínseca, orientación extrínseca, valor de la tarea, control sobre creencias y autoeficacia y correlaciona negativamente con ansiedad. La competencia correlaciona positivamente con orientación intrínseca, orientación extrínseca, valor de la tarea, control sobre creencias y autoeficacia. La buena voluntad correlaciona positivamente con orientación intrínseca, orientación extrínseca, valor de la tarea, control sobre creencias y autoeficacia y correlaciona negativamente con ansiedad. La confianza correlaciona positivamente con orientación intrínseca, orientación extrínseca, valor de la tarea, control sobre creencias y autoeficacia y correlaciona negativamente con ansiedad. En el modelo alternativo, la escala global de credibilidad correlaciona positivamente con orientación intrínseca, orientación extrínseca, valor de la tarea, control sobre creencias y autoeficacia y correlaciona negativamente con ansiedad. La competencia correlaciona positivamente con orientación intrínseca, orientación extrínseca, valor de la tarea, control sobre creencias y autoeficacia. La buena voluntad correlaciona positivamente con orientación intrínseca, orientación extrínseca, valor de la tarea, control sobre creencias y autoeficacia. La confianza correlaciona positivamente con orientación intrínseca, orientación extrínseca, valor de la tarea, control sobre creencias y autoeficacia y correlaciona negativamente con ansiedad. 
Como principal limitación del estudio, señalar que la muestra estuvo compuesta sólo por estudiantes universitarios. Consideramos que habría sido muy valioso utilizar participantes de distintos niveles educativos para disponer de una muestra que fuera lo más heterogénea posible. En este sentido, proponemos como líneas de investigaciones futuras determinar si la escala de credibilidad presenta resultados similares a los obtenidos en el presente estudio empleando participantes de diferentes niveles educativos como bachillerato o educación secundaria.

A pesar de esta limitación, consideramos que las versiones en español de la escala Source Credibility Measure obtenidas en nuestro estudio constituyen instrumentos fiables y válidos para medir la credibilidad del profesorado universitario. Creemos que ambos modelos pueden ser de utilidad para todos aquellos investigadores hispanohablantes interesados en analizar la credibilidad de los docentes en el ámbito universitario, teniendo importantes implicaciones prácticas en el ámbito académico debido al impacto de la credibilidad del profesorado en los procesos de enseñanzaaprendizaje (Finn et al., 2009).

\section{Referencias}

Al-Zoubi, Z. H. (2016). Student perceptions of college teacher misbehaviors and teacher credibility as perceived by Jordanian university students. Mediterranean Journal of Social Sciences, 7(2), 324-330. doi:10.5901/mjss.2016.v7n2p324

Applbaum, R. L., \& Anatol, K. W. (1973). Dimensions of source credibility: A test for reproducibility. Speech Monographs, 40(3), 230-237. doi:10.1080/03637757309375800

Baudhuin, E. S., \& Davis, M. K. (1972). Scales of the measurement of ethos: Another attempt. Speech Monographs, 39(4), 296-301. doi:10.1080/03637757209375769

Banfield, S. R., Richmond, V. P., \& McCroskey, J. C. (2006). The effect of teacher misbehaviors on teacher credibility and affect for the teacher. Communication Education, 55(1), 63-72. doi:10.1080/03634520500343400
Batista, J. M., \& Coenders, G. (2000). Modelos de ecuaciones estructurales. Madrid: La Muralla.

Beatty, M. J., \& Behnke, R. R. (1980). Teacher credibility as a function of verbal content and paralinguistic cues. Communication Quarterly, 28(1), 55-59. doi:10.1080/01463378009369358

Beatty, M. J., \& Zahn, C. J. (1990). Are student ratings of communication instructors due to "easy" grading practices?: An analysis of teacher credibility and student reported performance levels. Communication Education, 39(4), 275-282. doi:10.1080/03634529009378809

Brown, T. A. (2006). Confirmatory factor analysis for applied research. New York: Guildford Press.

Browne, M. W., \& Cudeck, R. (1992). Alternative ways of assessing model fit. Sociological Methods \& Research, 21(2), 230-258. doi:10.1177/0049124192021002005

Byrne, B. M. (2016). Structural equation modeling with AMOS. Basic concepts, applications, and programming $\left(3^{\text {rd }} \mathrm{Ed}\right.$.). New York and London: Routledge. doi:10.4324/9781315757421

Chory, R. M. (2007). Enhancing student perceptions of fairness: The relationship between instructor credibility and classroom justice. Communication Education, 56(1), 89105. doi:10.1080/03634520600994300

Cooper, L. (1935). The rhetoric of Aristotle. Quarterly Journal of Speech, 21(1), 10-19. doi:10.1080/00335633509380070

DiStefano, C. (2002). The impact of categorization with confirmatory factor analysis. Structural Equation Modeling, 9(3), 327-346. doi:10.1207/s15328007sem0903_2

Edwards, C., \& Myers, S. A. (2007). Perceived instructor credibility as a function of instructor aggressive communication. Communication Research Reports, 24(1), 4753. doi:10.1080/08824090601128141

Finn, A., \& Ledbetter, A. (2013). Teacher power mediates the effects of technology policies on teacher credibility. Communication Education, $\quad 62(1), \quad$ 26-47. doi:10.1080/03634523.2012.725132

Finn, A., Schrodt, P., Witt, P., Elledge, N., Jernberg, K., \& Larson, L. (2009). A meta 
analytical review of teacher credibility and its associations with teacher behaviors and student outcomes. Communication Education, 58(4), 516-537. doi:10.1080/03634520903131154

Frymier, A. B., \& Thompson, C. A. (1992). Perceived teacher affinity-seeking in relation to perceived teacher credibility. Communication Education, 41(4), 388-399. doi:10.1080/03634529209378900

Gaffney, J. D., \& Gaffney, A. L. H. (2016). Student satisfaction in interactive engagement-based physics classes. Physical Review Physics Education Research, 12(2), 1-17. doi:10.1103/physrevphyseducres.12.020125

Gil-Escudero, G., \& Martínez-Arias, M. R. (2001). Metodología de encuestas. En M. J. Navas (Ed.), Métodos, diseños y técnicas de investigación psicológica (pp. 379-436). Madrid: Universidad Nacional de Educación a Distancia.

Glascock, J., \& Ruggiero, T. (2006). The relationship of ethnicity and sex to professor credibility at a culturally diverse university. Communication Education, 55(2), 197-207. doi:10.1080/03634520600566165

Gómez-López, M., Granero-Gallegos, A., BaenaExtremera, A., Bracho-Amador, C., \& PérezQuero, F. J. (2015). Efectos de interacción de sexo y práctica de ejercicio físico sobre las estrategias para la disciplina, motivación y satisfacción con la educación física. Revista Iberoamericana de Diagnóstico y Evaluación - e Avaliação Psicológica, 40(2), 6-16.

Hambleton, R. K. (2004). Theory, methods, and practices in testing for the 21 st century. Psicothema, 16(4), 696-701.

Hendrix, K. G. (1998). Student perceptions of the influence of race on professor credibility. Journal of Black Studies, 28(6), 738-763. doi:10.1177/002193479802800604

Henning, Z. (2010). Teaching with style to manage student perceptions: The effects of socio-communicative style and teacher credibility on student affective learning. Communication Research Reports, 27(1), 5867. doi:10.1080/08824090903526471

Heyman, S. (1992). A study of Australian and Singaporean perceptions of source credibility. Communication Research Reports, 9(2), 137150. doi:10.1080/08824099209359906
Holgado-Tello, F. P., Chacón-Moscoso, S., Barbero-García, I., \& Vila-Abad, E. (2010). Polychoric versus Pearson correlations in exploratory and confirmatory factor analysis of ordinal variables. Quality \& Quantity, 44(1), 153-166. doi:10.1007/s11135-008-9190-y

Hovland, C. I., Janis, I. L., \& Kelley, H. H. (1954). Communication and persuasion. American Sociological Review, 19(3), 355357. doi: $10.2307 / 2087772$

Hu, L.T., \& Bentler, P. M. (1999). Cutoff criteria for fit indexes in co-variance structure analysis: Conventional criteria versus new alternatives. Structural Equation Modeling: A Multidisciplinary Journal, 6(1), 1-55. doi:10.1080/10705519909540118

Johnson, S. D., \& Miller, A. N. (2002). A crosscultural study of immediacy, credibility, and learning in the U.S. and Kenya. Communication Education, 51(3), 280-292. doi:10.1080/03634520216514

King, S. W., Minami, Y., \& Samovar, L. (1985). A comparison of Japanese and American perceptions of source credibility. Communication Research Reports, 2(1), 7679.

Lavin, A. M., Davies, T. L., \& Carr, D. L. (2010). The impact of instructor attire on student perceptions of faculty credibility and their own resultant behavior. American Journal of Business Education, 3(6), 51-62. doi:10.19030/ajbe.v3i6.442

Leal-Soto, F., \& Alonso-Tapia, J. (2017). Cuestionario de Clima Motivacional de la Clase: Validez intercultural, intergénero, evolutiva y predictiva. Revista Iberoamericana de Diagnóstico y Evaluación - e Avaliação Psicológica, 45(3), 57-70. doi:10.21865/RIDEP45.3.05

Ledbetter, A., \& Finn, A. (2016). Why do students use mobile technology for social purposes during class? Modeling teacher credibility, learner empowerment, and online communication attitude as predictors. Communication Education, 65(1), 1-23. doi:10.1080/03634523.2015.1064145

Martin, M. M., Chesebro, J. L., \& Mottet, T. P. (1997). Students' perceptions of instructors' sociocommunicative style and the influence on instructor credibility and situational 
motivation. Communication Research Reports, $\quad$ 14(4), 431-440. doi:10.1080/08824099709388686

Martínez, J. R., \& Galán, F. (2000). Estrategias de aprendizaje, motivación y rendimiento académico en alumnos universitarios. Revista Española de Orientación y Psicopedagogía, 11(19), 35-50.

Martinez-Egger, A. D., \& Powers, W. G. (2007). Student respect for a teacher: Measurement and relationships to teacher credibility and classroom behavior perceptions. Human Communication, 10(2), 145-155.

Mazer, J. P., \& Stowe, A. (2016). Can teacher immediacy reduce the impact of verbal aggressiveness? Examining effects on student outcomes and perceptions of teacher credibility. Western Journal of Communication, $\quad 80(1), \quad 21-37$. doi:10.1080/10570314.2014.943421

McCroskey, J. C. (1966). Scales for the measurement of ethos. Speech Monographs, 33(1), 65-72. doi:10.1080/03637756609375482

McCroskey, J. C. (1968). An Introduction to rhetorical communication. Englewood Cliffs, NJ: Prentice-Hall.

McCroskey, J. C. (1992). An introduction to communication in the classroom. Edina, MN: Burgess International.

McCroskey, J. C., \& Teven, J. J. (1999). Goodwill: A reexamination of the construct and its measurement. Communication Monographs, $\quad 66(1), \quad$ 90-103. doi:10.1080/03637759909376464

McCroskey, J. C., Valencic, K. M., \& Richmond, V. P. (2004). Toward a general model of instructional communication. Communication Quarterly, 52(3), 197-210. doi:10.1080/01463370409370192

McCroskey, J. C., \& Young, T. J. (1981). Ethos and credibility: The construct and its measurement after three decades. Central States Speech Journal, 32(1), 24-34. doi:10.1080/10510978109368075

McDonald, R. P. (1999). Test theory: A unified treatment. Mahwah, NJ, Lawrence: Erlbaum Associates.

Miller, A., N., Katt, J. A., Brown, T., \& Sivo, S. A. (2014). The relationship of instructor selfdisclosure, nonverbal immediacy, and credibility to student incivility in the college classroom. Communication Education, 63(1), 1-16. doi:10.1080/03634523.2013.835054

Morris, T., Gorham, J., Cohen, S., \& Huffman, D. (1996). Fashion in the classroom: Effects of attire on student perceptions of instructors in college classes. Communication Education, 45(2), 135-148. doi:10.1080/03634529609379043

Myers, S. A. (2001). Perceived instructor credibility and verbal aggressiveness in the college classroom. Communication Reports, 18(4), 354-364. doi:10.1080/08824090109384816

Myers, S. A. (2004). The relationship between perceived instructor credibility in college student in-class and out-of-class communication. Communication Reports, 17(2), 129-137. doi:10.1080/08934210409389382

Myers, S. A., Brann, M., \& Members of Comm 600. (2009). College students' perceptions of how instructors establish and enhance credibility through self-disclosure. Qualitative Research Reports in Communication, 10(1), 9-16.

Nadler, M. K., \& Nadler, L. B. (2001). The roles of sex, empathy, and credibility in out-of-class communication between faculty and students. Women's Studies in Communication, 24(2), 241-261. doi:10.1080/07491409.2001.10162436

Okabe, P. (1983). Cultural assumptions of east and west: Japan and the United States. En W. Gudykunst (Ed.), Intercultural communication theory: Current perspectives (pp. 21-44). Beverly Hills, CA: Sage.

Patton, T. O. (1999). Ethnicity and gender: An examination of its impact on instructor credibility in the university classroom. The Howard Journal of Communications, 10(2), 123-144. doi:10.1080/106461799246852

Pogue, L., \& AhYun, K. (2006). The effect of teacher nonverbal immediacy and credibility on student motivation and affective learning. Communication Education, 55(3), 331-344. doi:10.1080/03634520600748623

Ramos, N. C., Baghurst, T. A., \& McCullick, B. A. (2016). Elementary students' perceptions of PE teacher credibility in Brazil. Research Quarterly for Exercise and Sport, 87, 106107.

Russ, T., Simonds, C., \& Hunt, S. (2002). Coming out in the classroom... an occupational 
hazard?: The influence of sexual orientation on teacher credibility and perceived student learning. Communication Education, 51(3),311-324. doi:10.1080/03634520216516

Santilli, V., Miller, A. N. \& Katt, J. (2011). A comparison of the relationship between instructor nonverbal immediacy and teacher credibility in Brazilian and US classrooms. Communication Research Reports, 28(3), 266-274. doi:10.1080/08824096.2011.588583

Schrodt, P. (2003). Students' appraisals of instructors as a function of students' perceptions of instructors' aggressive communication. Communication Education, 52(2), 106-121. doi:10.1080/03634520302468

Schrodt, P., Turman, P. D., \& Soliz, J. (2006). Perceived understanding as a mediator of perceived teacher confirmation and students' ratings of instruction. Communication Education, 55(4), 370-388. doi:10.1080/03634520600879196

Schrodt, P., \& Witt, P. L. (2006). Students' attributions of instructor credibility as a function of students' expectations of instructional technology use and nonverbal immediacy. Communication Education, 55(1), 1-20. doi:10.1080/03634520500343335

Schrodt, P., Witt, P. L., Turman, P. D., Myers, S., Barton, M. H., \& Jernberg, K. (2009). Instructor credibility as a mediator of instructors' prosocial communication behaviors and students' learning outcomes. Communication Education, 58(3), 350-371. doi:10.1080/03634520902926851

Schumacher, R. E., \& Lomax, R. G. (2004). A beginner's guide to structural equation modeling ( $2^{\text {nd }}$ Ed.). Mahwah, New Jersey: Lawrence Erlbaum Associates, Publishers. doi:10.4324/9781410610904

Sebastian, R., \& Bristow, D. (2008). Formal or informal? The impact of style of dressand forms of address on business students' perceptions of professors. Journal of Education for Business, 83(4), 196-201. doi:10.3200/joeb.83.4.196-201

Semlak, J. L., \& Pearson, J. C. (2008). Through the years: An examination of instructor age and misbehavior on perceived teacher credibility. Communication Research Reports, 25(1), 76-85. doi:10.1080/08824090701831867
Teven, J. J. (2001). The relationship among teacher characteristics and perceived caring. Communication Education, 50(2), 159-169. doi:10.1080/03634520109379241

Teven, J. J. (2007). Teacher caring and classroom behavior: Relationships with student affect and perceptions of teacher competence and trustworthiness. Communication Quarterly, 55(4), 433-450. doi:10.1080/01463370701658077

Teven, J. J., \& Herring, J. E. (2005). Teacher influence in the classroom: A preliminary investigation of perceived instructor power, credibility, and student satisfaction. Communication Research Reports, 22(3), 235-246. doi:10.1080/00036810500230685

Teven, J. J., \& Katt, J. (2016). Instructor Credibility. En P. Witt (Ed.), Communication and Learning (pp. 183-210). Handbook of communication science: De Gruyter. doi:10.1515/9781501502446-009

Teven, J. J., \& McCroskey, J. C. (1997). The relationship of perceived teacher caring with student learning and teacher evaluation. Communication Education, 46(1), 1-9. doi:10.1080/03634529709379069

Thweatt, K. S., \& McCroskey, J. C. (1998). The impact of teacher immediacy and misbehaviors on teacher credibility. Communication Education, 47(4), 348-358. doi:10.1080/03634529809379141

Tibbles, D., Richmond, V., McCroskey, J., \& Weber, K. (2008). Organizational orientations in an instructional setting. Communication Education, 57(3), 389-407. doi:10.1080/03634520801930095

Tuppen, C. J. S. (1974). Dimensions of communicator credibility: An oblique solution. Speech Monographs, 41(3), 253-260. doi:10.1080/03637757409375844

Wang, Z., Novak, H., Scofield-Snow, H., Traylor, S., \& Zhou, Y. (2015). Am I disclosing too much? Student perceptions of teacher credibility via Facebook. The Journal of Social Media in Society, 4(1), 5-37.

Whitehead, J. L. (1968). Factors of source credibility. Quarterly Journal of Speech, 54(1), 59-63. doi:10.1080/00335636809382870

Yoon, K., Kim, C. H., \& Kim, M. S. (1998). A cross-cultural comparison of the effects of source credibility on attitudes and behavioral 
intentions. Mass Communication \& Society, 1(3),

153-173. doi:10.1080/15205436.1998.9677854

Zhang, Q. (2009). Perceived teacher credibility and student learning: Development of a multicultural model. Western Journal of Communication, 73(3), 326-347. doi:10.1080/10570310903082073

Zhang, Q., Zhang, J., \& Castelluccio, A. (2011). A cross-cultural investigation of student resistance in college classrooms: The effects of teacher misbehaviors and credibility. Communication Quarterly, 59(4), 450-464. doi:10.1080/01463373.2011.597287 